\title{
A fuzzy pert approach to evaluate plant construction project scheduling risk under uncertain resources capacity
}

\author{
Hsian Jong Hsiau; Chun Wei R.Lin \\ National Yunlin University of Science \& Technology (TAIW AN) \\ g9421805@yuntech.edu.tw, lincwr@yuntech.edu.tw
}

Received September 2008

Accepted April 2009

\begin{abstract}
A plant construction project always involves lots of activities. Precise information about the activities duration is unfortunately unavailable due to the uncertain resources capacity. The fuzzy program evaluation and review technique (PERT) has been widely applied to solve the fuzzy project scheduling problem. This paper presents an extended fuzzy PERT approach with four major improvement aspects to support the construction project scheduling management: 1) Evaluate operation fuzzy times based on available working volumes, resources quantity and fuzzy capacity of resources, 2) Adopting a maximal $\alpha_{i}$-levelcut method to compare the fuzzy precedent activities times to determine the reasonable earliest starting times of each activity, 3) Using fuzzy algebra method instead of fuzzy subtraction method to compute the fuzzy latest starting times and 4) Developing a project scheduling risk index (PSRI) to assist the decision maker to evaluate the project scheduling risk. Simulations experiments are conducted and demonstrated satisfactory results.
\end{abstract}

Keywords: Fuzzy PERT approach, project scheduling, construction project, project management 


\section{Introduction}

The plant construction project scheduling is not easy to handle due to various uncertain factors. For example the resources capacity is an important uncertain. Its uncertainty will impact the project scheduling. In industrial practice the decision makers usually use crisp value to estimate the project time while they bid a potential project. But when they get the orders or contracts, frequently they can't complete construction on time and the resulting cost always exceeds original expectations. How to evaluate the construction project scheduling risk is an important problem.

Fuzzy PERT (program evaluation review techniques) has been widely used to describe the uncertain task durations and scheduling of real industrial practice in project management. There are vast literatures devoted to research about the fuzzy PERT theories and applications. Mon et al. (1995) applied fuzzy distributions on project management to analysis schedule and cost. Chanas, S. \& Zielinski, P. (2001) analysis critical patch in the network with fuzzy activity times. Dubois et al. (2003a) studied on latest starting times and floats in activity networks with illknown durations. Dubois et al. (2003b) also planed fuzzy scheduling with incomplete knowledge. Slyetsov et al. (2003) researched the fuzzy temporal characteristics of operations for project management based on the network models. Wang (1999) developed a fuzzy set approach to schedule product development projects with temporal information. Wang (2002) used a fuzzy project scheduling approach to minimize schedule risk for product development. Wang (2004) applied a genetic algorithm for solving the problem under the objective of maximizing the worst case scheduling. Nezhad et al. (2008) proposed a fuzzy number maximum operator approximation and its application in fuzzy shop scheduling. However, there are still several unsolved issues in fuzzy PERT applications:

- The operation time of each activity is seldom available even using fuzzy number in construction project. If decision makers directly assume operation times of activities to plan the scheduling of project, the result of scheduling may be imprecise. 
- There are many ranking methods of fuzzy numbers. However a suitable method to compute the earliest starting times of each activity in project network has not developed yet.

- Fuzzy subtraction method to compute the fuzzy latest starting times may get the unreasonable negative values of times.

- It is worth developing a project scheduling risk index (PSRI) to assist the decision maker to evaluate scheduling risk while they bid a potential construction project.

In coping with the aforementioned issues, this paper presents an extended fuzzy PERT approach with four major improvement aspects to support the project scheduling management: 1) Evaluate operation fuzzy times based on available working volumes, resources quantity and fuzzy capacity of resources, 2) Adopting a maximal $\alpha_{i}$-level cut method to compare the fuzzy precedent activities times to determine the reasonable earliest starting times of each activity, 3) Using fuzzy algebra method instead of fuzzy subtraction method to compute the fuzzy latest starting times and 4) Developing an index PSRI to assist the decision makers to evaluate the project scheduling risk.

In this plant construction project scheduling problem, major assumptions are made as follows:

- A project has items of $N$ activities

- The precedence or succeed relations between each activity are available

- Working volumes of each activity $W_{n}$ are available from bidding information

- Resources quantity $K_{n}$ for each activity is available

- Decision maker can get the information about the fuzzy working capacity of resources

- The fuzzy working capacity of resources for $n t h$ activity can be represented as a trapezoid fuzzy number (TFN) $\tilde{V}_{n}=\left(v_{n 1}, v_{n 2}, v_{n 3}, v_{n 4}\right)$

The membership of TFN $\tilde{V}_{n}$ is defined 


$$
\tilde{V}_{n}(x)=\left\{\begin{array}{l}
\mu_{\tilde{A}}^{L}=\left(x-v_{n 1}\right) /\left(v_{n 2}-v_{n 1}\right), v_{n 1} \leq x \leq v_{n 2} \\
\omega=1, v_{n 2} \leq x \leq v_{n 3} \\
\mu_{\tilde{A}}^{R}=\left(x-v_{n 4}\right) /\left(v_{n 3}-v_{n 4}\right), v_{n 3} \leq x \leq v_{n 4} \\
0, \text { otherwise }
\end{array}\right.
$$

$v_{n 1}$ : the most pessimistic fuzzy working capacity of resources

$\left[v_{n 2}, v_{n 3}\right]$ : the most possible fuzzy working capacity of resources

$v_{n 4}$ : the most optimistic fuzzy working capacity of resources

\section{Definitions of fuzzy pert operators}

The most often used operators for fuzzy PERT are addition, subtraction, maximum, minimum and ranking. Addition operator is applied to calculate the earliest completing times and overall project completing time. Subtraction operator is used to compute the latest starting and completing times. Maximum operator is applied for earliest starting times. Suppose two trapezoid fuzzy numbers are defined $\tilde{X}=\left[x_{1}, x_{2}, x_{3}, x_{4}\right]$ and $\tilde{Y}=\left[y_{1}, y_{2}, y_{3}, y_{4}\right]$. The most often used formulas of addition, subtraction, maximum and minimum are the following:

Addition: $\tilde{X} \oplus \tilde{Y}=\left[x_{1}+y_{1}, x_{2}+y_{2}, x_{3}+y_{3}, x_{4}+y_{4}\right]$

Subtraction: $\tilde{X}-\tilde{Y}=\left[x_{1}-y_{4}, x_{2}-y_{3}, x_{3}-y_{2}, x_{4}-y_{1}\right]$

Maximum: $\max \{\tilde{X}, \tilde{Y}\}=\left[\max \left\{x_{1}, y_{1}\right\}, \max \left\{\mathrm{x}_{2}, y_{2}\right\}, \max \left\{x_{3}, y_{3}\right\}, \max \left\{x_{4}, y_{4}\right\}\right]$

Minimum: $\min \{\tilde{X}, \tilde{Y}\}=\left[\min \left\{x_{1}, y_{1}\right\}, \min \left\{\mathrm{x}_{2}, y_{2}\right\}, \min \left\{x_{3}, y_{3}\right\}, \min \left\{x_{4}, y_{4}\right\}\right]$

In this paper a fuzzy algebra with $\alpha_{i}$-level cut method instead of fuzzy subtraction method is proposed to avoid the inflation and unreasonable negative completing time. Let $\tilde{Y}^{\alpha i} \oplus \tilde{Z}^{\alpha i}=\tilde{X}^{\alpha i}$ to find $\tilde{Z}^{\alpha i}$.

$$
\begin{gathered}
\tilde{Y}^{\alpha i} \oplus \tilde{Z}^{\alpha i}=\left[y_{L}^{\alpha i}+z_{L}^{\alpha i}, y_{R}^{\alpha i}+z_{R}^{\alpha i}\right]=\tilde{X}^{\alpha i}=\left[x_{L}^{\alpha i}, x_{R}^{\alpha i}\right], \forall \alpha i \in[0,1] \\
\tilde{Z}^{\alpha i}=\left[x_{L}^{\alpha i}-y_{L}^{\alpha i}, x_{R}^{\alpha i}-y_{R}^{\alpha i}\right]
\end{gathered}
$$


The result of $\max \{\tilde{X}, \tilde{Y}\}$ and $\min \{\tilde{X}, \tilde{Y}\}$ from previous Maximum operator formula is still a TFN, but the real membership of $\max \{\tilde{X}, \tilde{Y}\}$ and $\min \{\tilde{X}, \tilde{Y}\}$ may be not TFN any more. In this paper, Max $\alpha_{i}$-level cut method is proposed to obtain more reasonable membership of earliest starting times and latest completing times. The operators are defined:

$$
\begin{gathered}
\max \left\{\tilde{X}^{\alpha i}, \tilde{Y}^{\alpha i}\right\}=\left[\max \left\{x_{L}^{\alpha i}, y_{L}^{\alpha i}\right\}, \max \left\{x_{R}^{\alpha i}, y_{R}^{\alpha i}\right\}\right], \forall \alpha i \in[0,1] \\
\min \left\{\tilde{X}^{\alpha i}, \tilde{Y}^{\alpha i}\right\}=\left[\min \left\{x_{L}^{\alpha i}, y_{L}^{\alpha i}\right\}, \min \left\{x_{R}^{\alpha i}, y_{R}^{\alpha i}\right\}\right], \forall \alpha i \in[0,1]
\end{gathered}
$$

Suppose $M$ fuzzy numbers are $\tilde{A}_{m}, m=1,2, \ldots, M$, the membership values of $\tilde{A}_{m}$ at $\alpha_{i}$-level cut will be $\tilde{A}_{m}^{\alpha i}=\left[A_{m L}^{\alpha i}, A_{m R}^{\alpha i}\right], \forall i \in[0,1], m=1,2, \ldots, M$.

Comparing all $\alpha_{i}$-level cut values of $\tilde{A}_{m}$ fuzzy numbers at $\alpha_{i}$ level and taking the maximum value at each level cut. The set of maximum value is $\tilde{R}^{\alpha i}=\left[\tilde{R}_{L}^{\alpha i}, \tilde{R}_{R}^{\alpha i}\right]=\max _{\substack{m \in\{1,2, \ldots, M\} \\ \forall i \in P}}\left[\tilde{A}_{m L}^{\alpha i}, \tilde{A}_{m L}^{\alpha i}\right]$. An example of Max $\alpha_{i}$-level result $\tilde{R}^{\alpha i}$ with three fuzzy numbers is illustrated as:

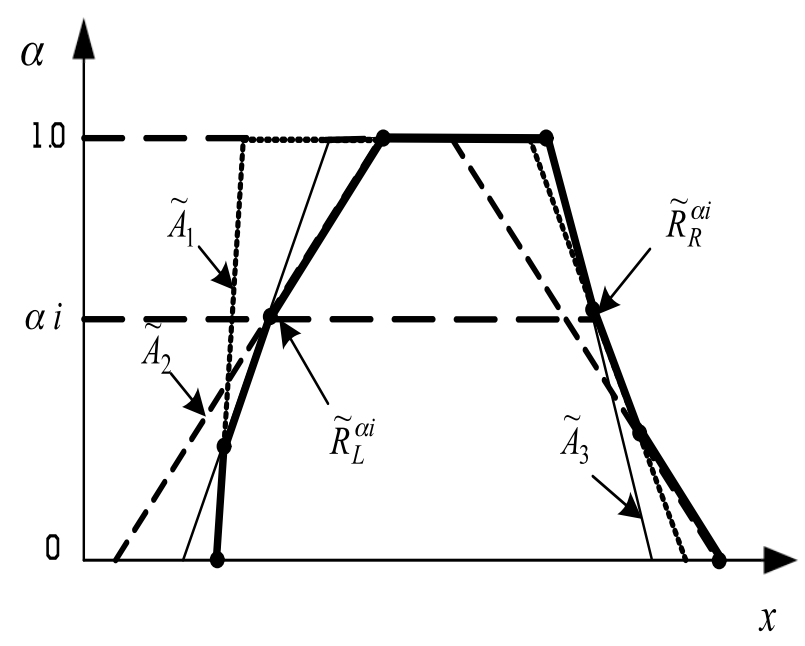

Figure 1. "Maximum result of $\tilde{R}^{\alpha i}=\max _{\forall i \in p}\left[\tilde{A}_{1}^{\alpha i}, \tilde{A}_{2}^{\alpha i}, \tilde{A}_{3}^{\alpha i}\right]$ ”. 


\section{Ranking:}

Ranking fuzzy numbers applied in fuzzy PERT is used to determine the earliest starting time. Techniques for ranking fuzzy numbers are abundant in the literature. Let $\tilde{X}=\left[x_{1}, x_{2}, x_{3}, x_{4}\right]$ and $\tilde{Y}=\left[y_{1}, y_{2}, y_{3}, y_{4}\right]$ are two trapezoid fuzzy numbers. If $x_{1} \leq y_{1}, x_{2} \leq y_{2}, x_{3} \leq y_{3}$ and $x_{4} \leq y_{4}$, the ranking of $\tilde{X}$ and $\tilde{Y}$ is said that $\tilde{X}$ is strongly greater than $\tilde{Y}$. If one of these four inequalities is not true, the comparison rule has to take the advantage of weak comparison rule (WCR). The rule is so-called defuzzifying ranking method.

But using defuzzifying ranking method to obtain the maximum fuzzy number, the comparison result is the maximum value of fuzzy numbers which participating in comparison. It can't fully express the character of two or more fuzzy numbers. In this studying, we propose Max $\alpha_{i}$-levelcut method. The result of using this method seems more reasonable than defuzzifying ranking method.

\section{The extended fuzzy pert approach}

- In this section we use the extended fuzzy PERT approach to create the computing procedure model for plant construction project scheduling and risk index. The computing procedure model are as follows:

Step1. Input parameters of project. The parameters are including:

- Items of project activity $N$

- Precedent or succeed relations between activities

- Working volumes of each activity $W_{n}$

- $\quad$ Fuzzy capacity of resources for each activity $\tilde{V}_{n}=\left(v_{n 1}, v_{n 2}, v_{n 3}, v_{n 4}\right)$

- Resources quantity for each activity $K_{n}$

- Overall project contract time $T_{c o n}$ and the maximum PSRI $\lambda_{\max }$ which decision maker can accept

- Numbers of $\alpha_{i}$-level cut $p$ 
Step 2. Compute fuzzy operation time of each activity

It is hard to directly know the operation times of activities in plant construction project. Each fuzzy operation time of activity in project network need to be determined by fuzzy divided method based on working volumes, resources quantity and fuzzy capacity of resources. The proposed formula is shown

$$
\tilde{A}_{n}=\left[a_{n 1}, a_{n 2}, a_{n 3}, a_{n 4}\right]=\frac{W_{n}}{K_{n}}\left[\frac{1}{v_{n 4}}, \frac{1}{v_{n 3}}, \frac{1}{v_{n 2}}, \frac{1}{v_{n 1}}\right]
$$

Step 3. Compute the memberships of fuzzy operation time for each activity at $\alpha_{i}$-level cut

In this paper, the $\operatorname{Max} \alpha_{i}$-level cut method to compute the scheduling times for each activity is proposed. Therefore, the membership values at $\alpha_{i}$-level cut for fuzzy operation time of each activity need to be computed. The membership values at $\alpha_{i}$-level cut are computed base on $\Delta \alpha$ value. Suppose decision maker set numbers of $\alpha_{i}$-level cut $p$, then $\Delta \alpha=\frac{1}{p}$.

From step 2, the fuzzy operation time of each activity $\tilde{A}_{n}=\left[a_{n 1}, a_{n 2}, a_{n 3}, a_{n 4}\right]$ is obtained. The membership values of each activity at $\alpha_{i}$-levelcut is $\tilde{A}_{n}^{\alpha i}=\left[A_{n L}^{\alpha i}, A_{n R}^{\alpha i}\right]$.

$$
\begin{gathered}
\text { Where } A_{n L}^{\alpha i}=a_{n 1}+\left(a_{n 2}-a_{n 1}\right) \times \alpha_{i} \\
\begin{array}{c}
A_{n R}^{\alpha i}=a_{n 4}-\left(a_{n 4}-a_{n 3}\right) \times \alpha_{i} \\
\alpha_{i}=\Delta \alpha \times i \\
\forall i \in P=\{0,1,2, \ldots, p\}
\end{array}
\end{gathered}
$$

Step 4. Compute the earliest starting fuzzy time for each activity ( $\widetilde{E} S$ )

Fuzzy PERT usually uses forward method to compute the earliest starting fuzzy time for each activity in network. The computing procedure of earliest starting fuzzy times for each activity are as bellow: 
Suppose there are $N$ items in total project network, the first starting item is $\sigma$ and last completing item is $\omega$, the earliest starting time with trapezoid fuzzy number for item $\sigma$ is $\tilde{E} S_{\sigma}=(0,0,0,0)$

The earliest starting fuzzy time for each activity $n$ is:

$$
\tilde{E} S_{n}=\max _{m \in \operatorname{pred}(n)}\left(\tilde{E} S_{m}+\tilde{A}_{m}\right)
$$

It shows the earliest starting fuzzy time for activity $n$ is the maximum fuzzy time of all precedent activities $m$ completing fuzzy times. In this step, we propose the Max $\alpha_{i}$-level cut method to calculate the membership of $\max _{m \in \operatorname{pred}(n)}\left(\tilde{E} S_{m}+\tilde{A}_{m}\right)$. If decision maker sets numbers of $\alpha_{i}$-level cut are $p, \Delta \alpha$ value is decided, the set of $\alpha_{i}$-level cut is $\alpha_{P}=\{\alpha i \mid \forall i \in P\}$.

From above computing procedure of Max $\alpha_{i}$-level cut, in this paper, we use it to compute the earliest starting fuzzy time for each activity and get the result:

$$
\tilde{E} S_{n}=\left[\max _{m \in \operatorname{pred}(n)}\left(E S_{m L}^{\alpha i}+A_{m L}^{\alpha i}\right), \max _{m \in \operatorname{pred}(n)}\left(E S_{m R}^{\alpha i}+A_{m R}^{\alpha i}\right)\right], \forall i \in P
$$

Step 5. Compute the earliest completing fuzzy time for each activity ( $\widetilde{E} C$ )

Slyeptsov et al. (2003) applied the equation $\tilde{E} C_{n}=\tilde{E} S_{n} \oplus \tilde{A}_{n}, n \in R$, to compute the earliest completing fuzzy time for activity $n$. The earliest completing fuzzy time for $n$ activity at $\alpha_{i}$-level cut can be written based on Max $\alpha_{i}-$ level method.

$$
\tilde{E} C_{n}=\left[\left(E S_{n L}^{\alpha i}+A_{n L}^{\alpha i}\right),\left(E S_{n R}^{\alpha i}+A_{n R}^{\alpha i}\right)\right], \forall i \in P
$$

Step 6. Compute the overall completing fuzzy time of total project ( $\widetilde{T} F$ )

The overall completing fuzzy time of total project is denoted $\widetilde{T} F$. Base on the equation $\widetilde{E} C_{n}=\widetilde{E} S_{n} \oplus \widetilde{A}_{n}$, the overall completing fuzzy time of total project $\widetilde{T} F$ will be equal to $\widetilde{E} C_{\omega}$, where $\omega$ is last completing item. Therefore, $\widetilde{T} F=\widetilde{E} C_{\omega}=\widetilde{E} S_{\omega} \oplus \widetilde{A}_{\omega}$. Using Max $\alpha_{i}$-level method, we can get:

$$
\tilde{T} F=\left[\left(E S_{\omega L}^{\alpha i}+A_{\omega L}^{\alpha i}\right),\left(E S_{\omega R}^{\alpha i}+A_{\omega R}^{\alpha i}\right)\right]=\left[E C_{\omega L}^{\alpha i}, E C_{\omega R}^{\alpha i}\right], \forall i \in P
$$


Step 7. Compute the latest completing fuzzy time for each activity ( $\widetilde{L} C$ )

To avoid the fuzzy number extending and unreasonable negative value after fuzzy number substraction operator, fuzzy algebra method is proposed to instead of fuzzy substraction method for computing the fuzzy latest dates of each activity.

$$
\begin{gathered}
\tilde{L} C_{\omega}=\left[L C_{\omega L}^{\alpha i}, L C_{\omega R}^{\alpha i}\right], \forall i \in P=\tilde{E} C_{\omega} \\
\tilde{L} C_{n}=\tilde{L} S_{n} \oplus \tilde{A}_{n}=\min _{m \in \operatorname{Succ}} \tilde{L} S_{m} \\
=\left[\min _{m \in \operatorname{Succ}(n)} L S_{m L}^{\alpha i}, \min _{m \in \operatorname{Succ}(n)} L S_{m L}^{\alpha i}\right], \forall i \in P \\
=\left[\min _{m \in \operatorname{Succ}(n)}\left(L C_{m L}^{\alpha i}-A_{m L}^{\alpha i}\right), \min _{m \in \operatorname{Succ}(n)}\left(L C_{m R}^{\alpha i}-A_{m R}^{\alpha i}\right)\right], \forall i \in P
\end{gathered}
$$

Step 8. Compute the latest starting fuzzy time for each activity ( $\widetilde{L} S$ )

Here, also use fuzzy algebra method to compute the latest starting fuzzy time for each activity. The computing procedures are these:

From step 6, we have computed the overall completing fuzzy time of total project $\widetilde{T} F=\widetilde{E} C_{\omega}$. The earliest and latest completing fuzzy time is same for last item activity $\omega$ in project. The latest completing fuzzy time last item activity $\omega$ is $\widetilde{L} C_{\omega}$, $\widetilde{L} C_{\omega}=\widetilde{T} F=\widetilde{E} C_{\omega}$.

Based on the formula of forward method: $\tilde{L} S_{n} \oplus \tilde{A}_{n}=\min _{m \in \text { Succ }} \tilde{L} S_{m}$

Base on $\alpha_{i}$-level cut method, using fuzzy algebra method to compute the latest starting fuzzy time for each activity $\widetilde{L} S_{n}$. The computing procedure is as following.

$$
\begin{gathered}
\tilde{A}_{n}=\left[A_{n L}^{\alpha i}, A_{n R}^{\alpha i}\right], \forall i \in P \\
\min _{m \in \operatorname{Succ}} \tilde{L} S_{m}=\left[\min _{m \in \operatorname{Succ}(n)} L S_{m L}^{\alpha i}, \min _{m \in \operatorname{Succ}(n)} L S_{m R}^{\alpha i}\right], \forall i \in P \\
=\tilde{L} C_{n}=\left[L C_{n L}^{\alpha i}, L C_{n R}^{\alpha i}\right], \forall i \in P \\
\tilde{L} S_{n} \oplus \tilde{A}_{n}=\left[\min _{m \in \operatorname{Succ}(n)} L S_{m L}^{\alpha i}, \min _{m \in \operatorname{Succ}(n)} L S_{m R}^{\alpha i}\right], \forall i \in P
\end{gathered}
$$




$$
\begin{gathered}
\tilde{L} S_{n}=\left[\min _{m \in \operatorname{Succ}(n)}\left(L S_{m L}^{\alpha i}-A_{n L}^{\alpha i}\right), \min _{m \in \operatorname{Succ}(n)}\left(L S_{m R}^{\alpha i}-A_{n R}^{\alpha i}\right)\right], \forall i \in P \\
=\left[L C_{n L}^{\alpha i}-A_{n L}^{\alpha i}, L C_{n R}^{\alpha i}-A_{n R}^{\alpha i}\right], \forall i \in P
\end{gathered}
$$

\section{Step 9. Compute PSRI}

The definition of PSRI is the possibility which overall project completing fuzzy time is longer than project contract time. The relationship between the overall project completing fuzzy time and project contract time is shown in figure 2 . In step 6 , we have derived the overall project completing fuzzy time $\widetilde{T} F$. If project contract time is a crisp value $T_{c o n}$, the PSRI $\lambda\left(\tilde{T} F, T_{c o n}\right)$ can be computed as below.

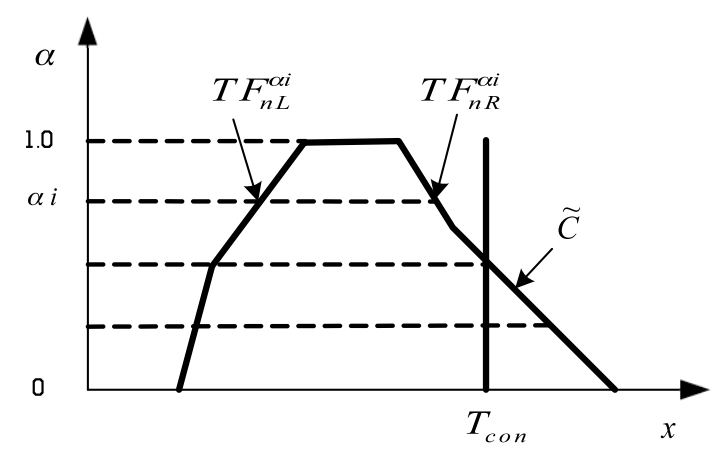

Figure 2. "The overall project completing fuzzy time vs. project contract time $T_{c o n}$ ".

$$
\begin{aligned}
& \text { If } T F_{n R}^{\alpha i}<T_{c o n}, D\left(\tilde{T} F^{\alpha i}>T_{c o n}\right)=0, D\left(\tilde{T} F^{\alpha i}<T_{c o n}\right)=1 \\
& \text { If } T F_{n L}^{\alpha i}>T_{c o n}, D\left(\tilde{T} F^{\alpha i}>T_{c o n}\right)=1, D\left(\tilde{T} F^{\alpha i}<T_{c o n}\right)=0 \\
& \text { If } T F_{n L}^{\alpha i}<T_{c o n}<T F_{n R}^{\alpha i}, D\left(\tilde{T} F^{\alpha i}>T_{c o n}\right)=\frac{T F_{n R}^{\alpha i}-T_{c o n}}{\left(T F_{n R}^{\alpha i}-T F_{n L}^{\alpha i}\right)} \\
& \qquad D\left(\tilde{T} F>T_{c o n}\right)+D\left(\tilde{T} F<T_{c o n}\right)=1 \\
& \text { PSRI } \lambda\left(\tilde{T} F, T_{c o n}\right)=D\left(\tilde{T} F>T_{c o n}\right)=\frac{\sum_{i=0}^{p} D\left(\tilde{T} F^{\alpha i}>T_{c o n}\right)}{p}
\end{aligned}
$$

The accuracy of $\lambda\left(\tilde{T} F, T_{c o n}\right)$ is relative to the number of $\alpha_{i}$-level cut $p$. 
If $\Delta \lambda=\left|\frac{\lambda_{p}-\lambda_{2 p}}{\lambda_{m}}\right|<\varepsilon$, finish computing PSRI $\lambda$, otherwise use the policy of increasing number $p$ to $2 p$ and repeat to compute $\lambda$. Were $\varepsilon$ is the accuracy of PSRI. If $\lambda \leq \lambda_{\max }$, potential contract time is acceptable, otherwise reject contract time.

Step 10. Output the computing results.

\section{Simulation experiments and performance evaluations}

For comparing the performance of computing results of PSRI and CPU times computed by $\operatorname{Max} \alpha_{i}$-level and defuzzifying ranking method individually, the varied factor of construction projects are conducted to simulate experiments. The hypothesis testing is used to confirm whether the result is significant.

\subsection{Experiment design}

In simulation experiments, there are two variable factors with two levels. Two variable factors are the number of activities ( 7 vs. 21) and the fuzzy time of resource capacity for each activity. Two levels are large or small for the number of activities and short or long for resource capacity of activity. The large or small number of activities is to describe the project complicated degree. The short or long fuzzy time for resource capacity of activity is to express the resources ability.

In order to process the simulation experiment for different conditions, the different variable factors and levels are grouped. Four groups of experiment shown as table 1 and figure 3 will be simulated. The overall project completing fuzzy time and PSRI are computed by Max $\alpha_{i}$-level and defuzzifying ranking method individually.

\begin{tabular}{|c|c|c|c|}
\hline Test & $n$ & $\widetilde{V}_{n}$ & $C$ \\
\hline 1 & 7 & Short & 30 \\
\hline 2 & 7 & Short & 30 \\
\hline 3 & 21 & Long & 90 \\
\hline 4 & 21 & Long & 90 \\
\hline
\end{tabular}

Table 1. "Simulation environments". 
The WCR that uses the middle of $\alpha$-cuts proposed by Dubois et al. (2000) seems to be the simplest one. A TFN $\tilde{X}=\left[x_{1}, x_{2}, x_{3}, x_{4}\right]$ through the defuzzifying operator, the result is $D F(\tilde{X})=\frac{1}{4}\left[x_{1}+x_{2}+x_{3}+x_{4}\right]$. This defuzzifying ranking method is used to compare Max $\alpha_{i}$-level method.

The fuzzy time of resource capacity for each activity is $\tilde{V}_{n}=\left[v_{n 1}, v_{n 2}, v_{n 3}, v_{n 4}\right], n$ is the item of activity. The values of $v_{n 1}, v_{n 2}, v_{n 3}, v_{n 4}$ can be generated by following equation during fuzzy interval in simulation program. In experiment, the short or long fuzzy times of resource capacity for activities are controlled by $\mathrm{C}$ value.

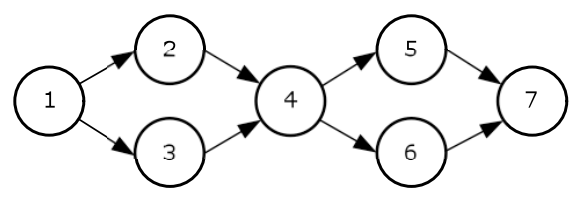

(a) Small project

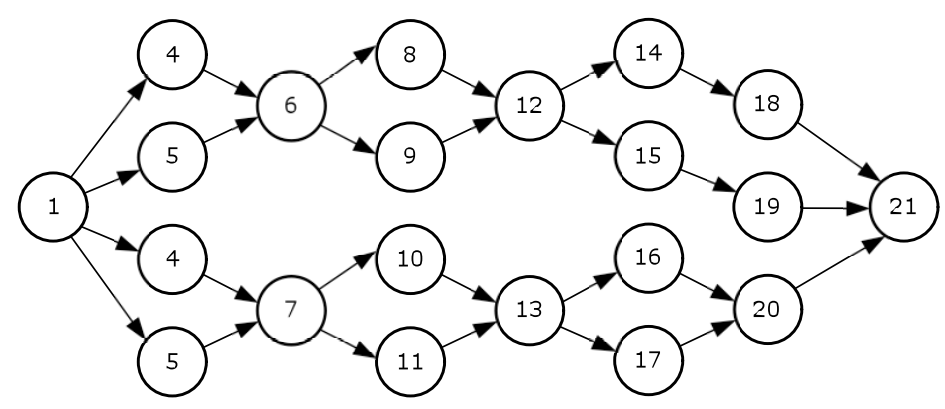

(b) Large project

Figure 3. "Project network".

$$
\begin{gathered}
v_{n 2}=C \times \text { Uniform }\left(m_{2}, 1.0\right), v_{n 1}=v_{n 2} \times \text { Uniform }\left(m_{1}, 1.0\right) \\
v_{n 3}=C \times \text { Uniform }\left(1.0, m_{3}\right), v_{n 4}=v_{n 3} \times \text { Uniform }\left(1.0, m_{4}\right)
\end{gathered}
$$

Where $v_{n 1} \leq v_{n 2} \leq v_{n 3} \leq v_{n 4}$ and $m_{1}=0.5, m_{2}=0.8, m_{3}=1.2, m_{4}=1.5$.

\subsection{Simulation results}

The simulation results of PSRI and CPU time are obtained from experiment by Max $\alpha_{i}$-level method and defuzzifying ranking method. The PRSI results are shown as 
in table 2 and all $P$-value are less than the level of significance 0.05 . The values of test statistics are all in the rejection region. Mean of PSRI of $\operatorname{Max} \alpha_{i}$-level is higher than defuzzifying ranking.

Also, the testing results of CPU times are shown as in table 3 and $P$-value are all less than the level of significance 0.05. The values of test statistics are all in the rejection region. The CPU time mean of $\operatorname{Max} \alpha_{i}$-level method is shorter than defuzzifying ranking method in four groups of experiment.

\begin{tabular}{|c|c|c|c|c|c|c|}
\hline \multirow{2}{*}{ Exp. item } & \multicolumn{4}{|c|}{ Mean of project risk level index } & \multicolumn{2}{c|}{ Testing result } \\
\cline { 2 - 5 } & $M A X \alpha_{i}-$ level method & Defuzzifying method & \multirow{2}{*}{$P$-value } & $H_{0}: \mu_{0}=\mu_{1}$ \\
\cline { 2 - 5 } & Mean $\mu_{0}$ & Std dev. & Mean $\mu_{1}$ & Std dev. & & \\
\hline 1 & 0.1614 & 0.0250 & 0.1384 & 0.0349 & 0.000 & Rejection \\
\hline 2 & 0.1614 & 0.0250 & 0.1384 & 0.0349 & 0.000 & Rejection \\
\hline 3 & 0.1462 & 0.0142 & 0.1239 & 0.0256 & 0.000 & Rejection \\
\hline 4 & 0.1462 & 0.0142 & 0.1219 & 0.0273 & 0.000 & Rejection \\
\hline
\end{tabular}

Table 2. "Mean of PSRI“.

\begin{tabular}{|c|c|c|c|c|c|}
\hline \multirow{2}{*}{ Exp. item } & \multicolumn{4}{|c|}{ CPU time mean } & Test results \\
\cline { 2 - 5 } & MAX $\alpha_{i}$-level method & Defuzzifying method & \multirow{2}{*}{ P - value } \\
\cline { 2 - 5 } & Mean $\mu_{2}$ & Std dev. & Mean $\mu_{3}$ & Std dev. & \\
\hline 1 & 0.3469 & 0.0652 & 0.4329 & 0.0875 & 0.000 \\
\hline 2 & 0.2282 & 0.0612 & 0.2666 & 0.0939 & 0.004 \\
\hline 3 & 0.3913 & 0.0634 & 0.9885 & 0.1659 & 0.000 \\
\hline 4 & 0.3746 & 0.0585 & 0.9543 & 0.1646 & 0.000 \\
\hline
\end{tabular}

Table 3. "Mean of CPU time".

\section{An example of petrochemical plant construction project}

An example of petrochemical plant construction project is illustrated as table 4. The project network is shown as figure 4. Parameters of problem are given: $T_{c o n}=360, \lambda_{\max }=0.1, p=10, \varepsilon=0.01$.

The overall project fuzzy time vs. contract time is illustrated as figure 5 . The membership of $\widetilde{T F}$ and the index of scheduling risk $\lambda$ are obtained.

$$
\begin{gathered}
\tilde{T} F^{\alpha i}=[291.3,293.4,295.6,297.7,299.9,302.6,305.8,308.9,312.4,316.5,320.5, \\
339.5,343.7,347.7,352.0,356.2,360.3,364.5,369.9,375.9,381.9,387.8]
\end{gathered}
$$


$\lambda=\lambda_{80}=0.0810, \lambda<\lambda_{\max }$, decision maker accept project contract time.

\begin{tabular}{|c|c|c|c|c|c|c|c|c|c|c|c|c|}
\hline \multirow{2}{*}{$n$} & \multirow{2}{*}{ Activity description } & \multirow{2}{*}{$W_{n}$} & \multicolumn{4}{|c|}{$\widetilde{V}_{n}=\left(v_{1}, v_{2}, v_{3}, v_{4}\right)$} & \multirow{2}{*}{$K_{n}$} & \multirow{2}{*}{$\begin{array}{l}\text { Precedence } \\
\text { item }\end{array}$} & \multicolumn{4}{|c|}{$\tilde{A}_{n}=\left(a_{n}, b_{n}, c_{n}, d_{n}\right)$} \\
\hline & & & $v_{1}$ & $v_{2}$ & $v_{3}$ & $v_{4}$ & & & $a_{n}$ & $b_{n}$ & $c_{n}$ & $d_{n}$ \\
\hline 1 & Piling & 480 & 24 & 25 & 26 & 27 & 1 & None & 18 & 18 & 19 & 20 \\
\hline 2 & Foundation & 4,800 & 30 & 30 & 32 & 33 & 3 & 1 & 48 & 50 & 53 & 53 \\
\hline 3 & Steel structure Fab. & 3,200 & 26 & 28 & 30 & 32 & 4 & None & 25 & 27 & 29 & 31 \\
\hline 4 & Steel structure Install & 3,200 & 30 & 32 & 33 & 34 & 2 & $2-3$ & 47 & 48 & 50 & 53 \\
\hline 5 & Equipment type 1 & 200 & 2 & 3 & 3 & 4 & 2 & 2 & 29 & 33 & 33 & 50 \\
\hline 6 & Equipment type 2 & 400 & 4 & 4 & 5 & 5 & 2 & 4 & 40 & 40 & 50 & 50 \\
\hline 7 & Equipment type 3 & 1,000 & 8 & 10 & 11 & 14 & 2 & 4 & 36 & 45 & 50 & 63 \\
\hline 8 & Piping Fabricated & 20,000 & 50 & 52 & 55 & 57 & 8 & None & 44 & 45 & 48 & 50 \\
\hline 9 & Piping Installation & 16,000 & 16 & 18 & 19 & 22 & 10 & $5-8$ & 73 & 84 & 89 & 100 \\
\hline 10 & Control room & 10,000 & 20 & 21 & 23 & 25 & 2 & 1 & 200 & 217 & 238 & 250 \\
\hline 11 & Equipment Flushing & 16 & 0.26 & 0.34 & 0.34 & 0.42 & 1 & 9 & 38 & 47 & 47 & 61 \\
\hline 12 & Piping Flushing & 3,600 & 60 & 70 & 80 & 85 & 1 & 9 & 42 & 45 & 51 & 60 \\
\hline 13 & Equipment Insulation & 8,500 & 75 & 85 & 90 & 120 & 2 & 9 & 35 & 47 & 50 & 57 \\
\hline 14 & Piping Insulation & 4,000 & 80 & 85 & 95 & 120 & 1 & 9 & 33 & 42 & 47 & 50 \\
\hline 15 & Instrument & 2,000 & 30 & 50 & 52 & 60 & 1 & 9 & 33 & 38 & 40 & 67 \\
\hline 16 & Electricity & 4,000 & 35 & 43 & 44 & 55 & 2 & 9 & 36 & 45 & 47 & 57 \\
\hline 17 & Test run & 16 & 0.5 & 0.6 & 0.6 & 0.7 & 1 & $10-16$ & 23 & 27 & 27 & 32 \\
\hline
\end{tabular}

Table 4. "Problem of petrochemical plant construction project".

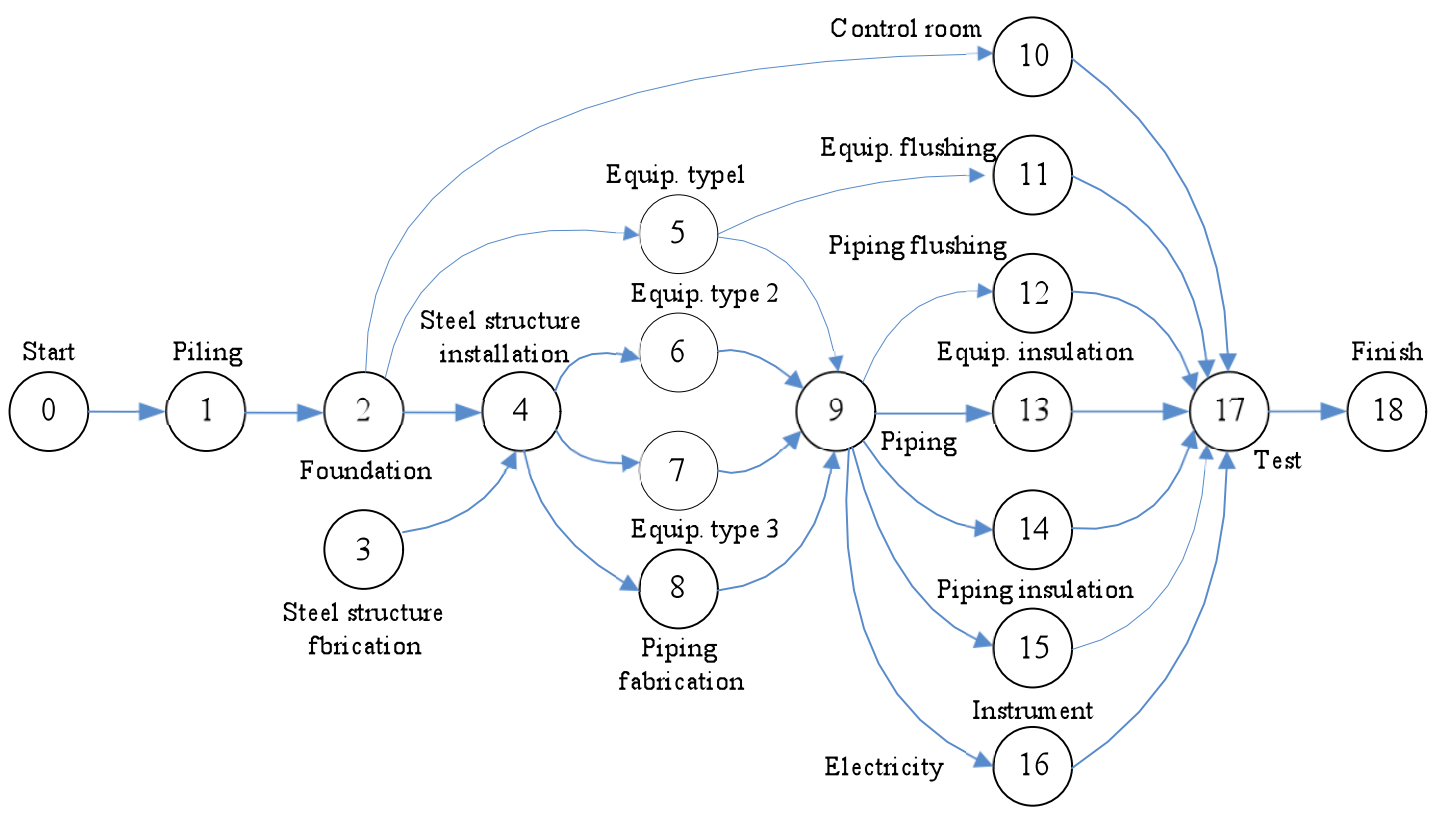

Figure 4. "Project network of petrochemical plant construction". 


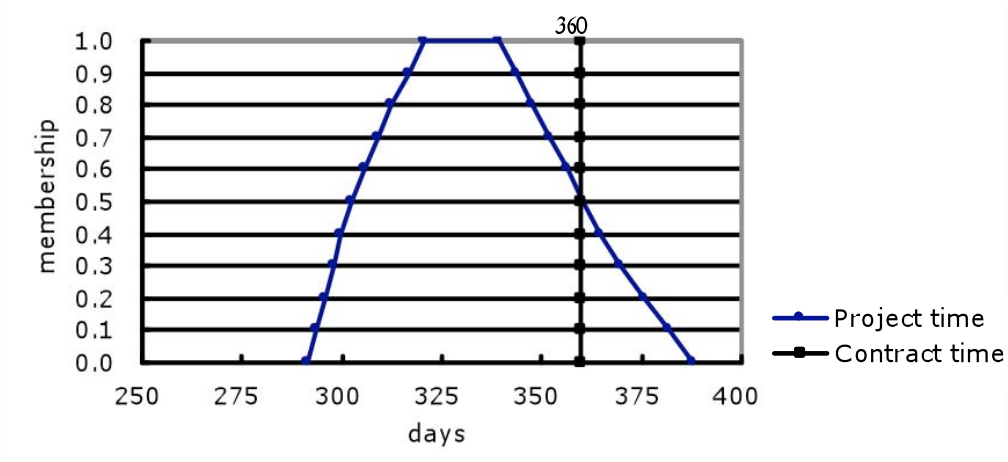

Figure 5. "Membership of overall project fuzzy time vs. contract time".

\section{Conclusions}

For managing the plant construction project scheduling and evaluating the risk of project contract time, we present an extended fuzzy PERT approach to solve the difficulties of traditional fuzzy PERT and the major achievements are as follows:

- Activity operation durations in project network are computed from task volumes, resources quantity and capacity of resources. An example of petrochemical plant construction project is demonstrated. The computing model is feasible and is proofed by simulation experiments.

- The proposed Max $\alpha_{i}$-level cut method outperformed the defuzzifying method to rank fuzzy number for determining the reasonable earliest starting time of each activity.

- Proposed fuzzy algebra method instead of fuzzy substraction method to compute the fuzzy latest times of each activity has avoided the fuzzy number extending and unreasonable negative value after fuzzy number substraction operator.

- Developing an index PSRI to assist the decision maker to evaluate scheduling risk is convenient. 


\section{References}

Carr, V., \& Tah, J.H.M., (2001). A fuzzy Approach to construction project risk assessment and analysis: construction project risk management system. Advance in Engineering Software, 32, 847-857.

Yates, J.K., \& Eskander, A. (2002). Construction total project management planning issues. Project Management J ournal, 33 (1), 37-48.

Chanas, S., \& Zielinski, P. (2001). Critical patch analysis in the network with fuzzy activity times. Fuzzy Sets and Systems, 122, 195-204.

Lootsma, F.A. (2001). Stochastic and fuzzy PERT. European Journal of Operational Research, 43(2), 174-183.

Mon, D.L., Cheng, C.H., \& Lu, H.C. (1995). Application of fuzzy distributions on project management. Fuzzy Sets and Systems, 73, 227-234.

Slyeptsov, A.I., \& Tyshchuk, T.A. (2003). Fuzzy temporal characteristics of operations for project management on the network models basis. European Journal of Operational Research, 147, 253-265.

Dubois, D., Fargier, H., \& Galvagnon, V. (2003a). On latest starting times and floats in activity networks with ill-known durations. European Journal of Operational Research, 147, 266-280.

Dubois, D., Fargier, H., \& Fortemps, P. (2003b). Fuzzy scheduling: Modeling flexible constraints vs. coping with incomplete knowledge. European Journal of Operational Research, 147, 231-252.

Wang, J. (1999). A fuzzy set approach to activity scheduling for product development. Journal of the Operation Research Society, 50, 1217-1228.

Wang, J. (2002). A fuzzy project scheduling approach to minimize schedule risk for product development. Fuzzy Sets and Systems, 127, 99-116.

Wang, J. (2004). A fuzzy robust scheduling approach for product development projects. European J ournal of Operational Research, 152, 180-194. 
Yoon K.P. (1996). A probabilistic approach to rank complex fuzzy numbers. Fuzzy Sets and Systems, 80, 167-176.

Nezhad, S.S., \& Assadi R.G. (2008). Preference ratio-based maximum operator approximation and its application in fuzzy flow shop scheduling. Applied Soft Computing, 8, 759-766.

Article's contents are provided on a Attribution-Non Commercial 3.0 Creative commons license. Readers are allowed to copy, distribute and communicate article's contents, provided the author's and Journal of Industrial Engineering and Management's names are included. It must not be used for commercial purposes. To see the complete license contents, please visit http://creativecommons.org/licenses/by-nc/3.0/. 